\title{
LIDERAZGO ESCOLAR Y CONFLICTOS SOCIOEDUCATIVOS. UN ESTUDIO EXPLORATORIO EN LICEOS PÚBLICOS CHILENOS $^{1}$
}

\author{
Cristóbal Villalobos ${ }^{2}$ \\ Javiera Peña ${ }^{3}$ \\ Elisa Aguirre ${ }^{4}$ \\ Magdalena Guerrero ${ }^{5}$
}

RESUMEN

La investigación busca estudiar los procesos de conflictividad educativa, dando cuenta de las estrategias y marcos de acción implementados por los directivos escolares en establecimientos públicos de educación media en Chile. Para ello, se realiza un estudio de caso en ocho liceos municipales, utilizando diversas herramientas cualitativas. Se obtienen tres principales resultados. En primer término, se observa que los conflictos que involucran como actor central a los estudiantes tienen mayoritariamente un origen relacionado con el contexto social, económico y cultural de estos, mientras que los conflictos que involucran a adultos tienen relación con problemáticas de la micropolítica y macropolítica escolar. En segundo término, y respecto de las dinámicas que adoptan los conflictos, se observa que en la mayoría de los casos estos tienden a transitar de conflictos cognitivos a conflictos afectivos, lo que conlleva que estos se personalicen y se visualicen como procesos improductivos para las escuelas. Finalmente, y en relación con las estrategias que utilizan los directivos para manejar los conflictos, se constata que estas tienden a estar basadas en la ejecución de reglamentos y protocolos de acción (especialmente cuando involucran a estudiantes), o bien, a estrategias de dilación o relativización de los conflictos (especialmente cuando refieren a profesores).

1 El presente artículo se enmarca en el estudio Conflictos socioeducativos y liderazgo escolar. Análisis y caracterización de los conflictos socioeducativos y las estrategias de gestión directivas en los liceos municipales de Chile, realizado por el Centro de Desarrollo del Liderazgo Educativo (CEDLE) de la Universidad Diego Portales. Adicionalmente, Cristóbal Villalobos agradece el patrocinio del Centro de Estudios del Conflicto y la Cohesión Social, COES (Conicyt/ Fondap/15130009).

2 Centro de Estudios de Políticas y Prácticas en Educación (CEPPE UC), Pontificia Universidad Católica de Chile, Santiago, Chile. Contacto: clvillal@uc.cl

3 Centro de Desarrollo del Liderazgo Educativo (CEDLE), Universidad Diego Portales, Santiago, Chile. Contacto: javiera.pena.fde@mail.udp.cl

4 Centro de Desarrollo del Liderazgo Educativo (CEDLE), Universidad Diego Portales, Santiago, Chile. Contacto: elisa.aguirre.p@gmail.com

5 Facultad de Educación, Universidad del Desarrollo, Santiago, Chile. Contacto: mm.guerrero@udd.cl 
Palabras clave: conflictos socioeducativos, directivos escolares, escuelas públicas, liderazgo, manejo y gestión de conflictos.

\section{SCHOOL LEADERSHIP AND SOCIOEDUCATIONAL CONFLICTS. AN EXPLORATORY STUDY IN CHILEAN PUBLIC HIGH SCHOOLS}

\section{ABSTRACT}

The research seeks to study the processes of educational conflict, giving an account of the strategies and frameworks of action implemented by school administrators in public secondary schools in Chile. In order to achieve this, a case study was carried out in eight public high schools, using several qualitative techniques. Three results are obtained. First, the conflicts that involve students as central actors mainly arise from their social, economic and cultural context. In contrast, conflicts involving adults are related to problems of micro-politics (school level) and macro-politics (field of education). The second result is related with the dynamics adopted by conflicts, where it is observed that, in most cases, conflicts tend to transition from cognitive conflicts to affective conflicts, which means that become personalized and viewed as unproductive processes for schools. Finally, and in relation to the strategies that school leaders use to handle the conflicts, it is verified that these differ depending on the actor involved. When they involve students, there are regulations and action protocols; whereas when teachers are involved, there are generally no regular channels to proceed, and conflicts tend to be avoided or not recognized.

Keywords: conflict management, leadership, public schools, school leaders, socio-educational conflicts.

\section{Introducción}

Uno de los aspectos que la literatura sociológica y educativa ha identificado como centrales en la configuración de la dinámica escolar es la existencia de conflictos, entendidos como aquellas formas de interacción social en las que al menos uno de los actores involucrados reconoce la existencia de incompatibilidades con otro actor (Glasl, 2004; Wagner, 2005). Así, diversos autores (Lima, 2001; Unline, Tschannen-Moran y Pérez, 2003) han destacado al conflicto como un elemento relevante en los procesos de institucionalización y configuración de la vida de las escuelas. Al mismo tiempo, se ha indicado que, al contrario de lo que propusieron las teorías funcionalistas, los conflictos pueden ser entendidos como un motor dinamizador de la realidad educativa (Córdoba-Alcaide, Ortega-Ruiz y Nail-Kröyer, 2016), señalándose incluso que las culturas escolares 
no florecen ni se desarrollan sin controversias internas (Lima, 2001). A pesar de su importancia, las investigaciones sobre conflictividad al interior de las escuelas en América Latina han tendido a concentrarse en algún tipo de conflicto o actor, siendo estudiados principalmente desde la perspectiva de la violencia y el clima escolar (Román y Murillo, 2011) o desde la lógica de las condiciones laborales de trabajo y los posibles conflictos que pueden producirse en este ámbito (Loyo, 2001).

Adicionalmente, es importante destacar que si bien durante las últimas décadas ha aumentado el interés por estudiar el liderazgo escolar y el rol de los directivos (Temperley y Stoll, 2011), reconociéndose el papel que estos juegan en la gestión y resolución de conflictos en las comunidades escolares (Handford $\&$ Leithwood, 2013; Tschannen-Moran, 2014) ${ }^{6}$, los estudios y discusiones respecto de este tema han tendido a ocupar un lugar secundario dentro de la producción intelectual en torno al liderazgo educativo (Gunter, 2016). Así, temáticas como el rol del liderazgo en los procesos pedagógicos y su eficacia en las escuelas (Day, Sammons, Hopkins, Leithwood \& Kington, 2008; Leithwood, Seashore, Anderson y Wahlstrom, 2004), la forma de ejercicio y distribución de las funciones del liderazgo dentro de las comunidades escolares (Spillane, 2012) o el análisis de la capacidad del liderazgo directivo en el fomento de la mejora escolar (Leithwood, Tomlinson \& Genge, 1996) han acaparado gran parte de la atención investigativa, relegando en alguna medida los debates en torno a los vínculos existentes entre el liderazgo educativo y los conflictos en las escuelas.

A diferencia de estas perspectivas, el presente estudio parte del supuesto de que los conflictos son un elemento consustancial a la organización escolar, siendo un momento crucial de la dinámica educativa en el que se visualizan disputas o controversias entre los distintos actores escolares, producidas tanto por causas externas como internas a esta organización. Desde esta óptica, los procesos

Una de las responsabilidades internacionalmente reconocidas de los directores es la de crear y promover las condiciones adecuadas para el buen desarrollo y funcionamiento de los establecimientos, creando procesos de armonía en la escuela (Centro de Estudios de Políticas y Prácticas en Educación, CEPPE, 2013). 
de manejo y gestión de los conflictos aparecen como un elemento central de análisis del espacio educativo. De manera genérica, estos procesos se entienden como todas aquellas acciones, estrategias y planes implementados con el objetivo de transformar los conflictos desde improductivos o negativos a positivos o productivos (Stewart, 2015). En general, se reconoce que un actor central en este proceso de gestión es el director o directivo escolar, entendiéndose, por lo mismo, el campo de las políticas e investigaciones sobre liderazgo educativo como un espacio fértil para el desarrollo de esta discusión (Gross \& Shapiro, 2013).

Considerando lo anterior, el objetivo de la investigación es estudiar los procesos de conflictividad educativa, dando cuenta de las estrategias y marcos de acción implementadas por los directivos escolares en establecimientos públicos de educación media en Chile. Si bien los estudios que vinculan los conflictos escolares y el liderazgo educativo han sido investigados en los últimos años a nivel internacional (Bartsch \& Cheurprakobkit, 2002; Coleman \& Fisher-Yoshida, 2004; Msila, 2012), tanto en América Latina como en Chile, las investigaciones en torno a esta materia son casi inexistentes, por lo que la presente exploración se entiende como un avance para el ámbito de la investigación educativa en general y del liderazgo educativo en específico.

Para responder a este objetivo, el artículo se organiza en cuatro apartados. En el primero se presentan los antecedentes conceptuales para entender el concepto ancla del estudio: los conflictos socioeducativos. Asimismo, se entregan antecedentes teóricos para comprender las dinámicas que adoptan estos en el espacio escolar y la relación entre el liderazgo escolar y el manejo de los conflictos al interior de las escuelas. El segundo apartado describe la metodología utilizada en la investigación, dando cuenta de la muestra seleccionada, las técnicas de recolección de información y el método de análisis utilizado. En la tercera sección se presentan los resultados del estudio, que se focalizan en tres aspectos: la descripción de los conflictos socioeducativos más frecuentes; la dinámica de producción de estos conflictos en el espacio escolar y; las principales estrategias de manejo y gestión de los conflictos utilizadas por los directivos escolares. 
Finalmente, se exponen las conclusiones obtenidas a partir de los hallazgos, dando cuenta también de las limitaciones del estudio y los desafíos para la política pública y educativa emergentes.

\section{Antecedentes conceptuales}

\subsection{Los conflictos socioeducativos como concepto emergente}

Cualquier conflicto social se produce cuando al menos dos actores sociales, ya sean individuos o grupos, se enfrentan implícita o explícitamente a causa de objetivos, formas de pensar, posiciones y/o valores que se visualizan como incompatibles (Glasl, 2004; Touraine, 2006; Wagner, 2005). En el espacio educativo, la noción de conflicto suele asociarse, al menos en América Latina, a las dinámicas de conflictividad que se producen entre estudiantes. Así, conceptos como violencia escolar, bullying y ciberbullying han tendido a operar como sinónimos del concepto de conflicto escolar en la investigación educativa (Madriaza y García, 2004; Quezada, Navarro y Mora, 2005). Este énfasis entregado a las relaciones entre estudiantes ha respondido a la comprensión de la escuela como el lugar de control de la violencia y socialización escolar, poniendo en el centro a los estudiantes como sujetos finales de recepción del proceso educativo, lo que en la práctica ha significado que las relaciones y conflictos entre otros actores escolares reciban menor atención.

En contraste con estas visiones, la presente investigación se basa en la noción de conflictos socioeducativos ${ }^{7}$, la que busca incluir dos aspectos de la dinámica escolar poco estudiados por la literatura de corriente principal en torno al tema. Por una parte, se busca hacer hincapié en la estrecha relación establecida entre la escuela, el campo educativo y el entorno político, cultural, social y económico en el que se desarrollan los establecimientos educativos (de ahí la noción de "socio"). Sin embargo, y de manera complementaria, se busca indicar que los conflictos que se producen al interior de las escuelas poseen una particularidad propia, que responde a su naturaleza organizativa

Para un desarrollo más acabado del concepto, ver Villalobos, Peña y Weinstein (2016). 
y que no son simplemente una reproducción de otros conflictos societales (de ahí la noción de "educativos").

Como es lógico, esta noción complementaria de conflictos se basa en la idea de la escuela como un espacio cerrado (que produce su propia dinámica conflictiva) y, a la vez, abierto (que desarrolla procesos de conflictividad a partir del contexto en el que se desenvuelve), siguiendo en este aspecto las discusiones desarrolladas por Bourdieu (1994) acerca de la teoría de los campos. Por ello, es posible distinguir, a lo menos en términos analíticos, tres fuentes $\mathrm{u}$ orígenes de este tipo de conflictos: aquellos que se producen al interior de la escuela; los que tienen su origen en el campo escolar; y aquellos que se originan en el contexto social, político, económico y cultural de la escuela.

Respecto de los primeros, Ball (1989) ya mostraba a fines de los ochenta cómo la disensión, la divergencia, la diversidad y la confrontación eran elementos inherentes a la institución educativa, dando cuenta de la necesidad de entender la micropolítica del espacio escolar y las múltiples relaciones entre los actores, las que se concebían como el "conjunto de estrategias con las cuales los individuos y grupos que se hallan en contextos educativos tratan de usar sus recursos de poder e influencia a fin de promover sus intereses" (Ball, 1989, p. 88).

Por otra parte, la segunda dimensión que configura los conflictos socioeducativos se relaciona con lo que se ha denominado macropolítica escolar (Jares, 1997), concepto utilizado para referirse a las relaciones establecidas entre el Estado, la administración del sistema escolar y la sociedad civil. Desde esta perspectiva, las escuelas se encuentran sujetas constantemente a las disputas, definiciones y directrices que provienen de este espacio (Jares, 1997; Mayer, 2009; Ruiz, 2006), y se ven influidas por tensiones, protestas, manifestaciones o incompatibilidades que, poseyendo un origen o motivación en una problemática del conjunto del campo, tienen una manifestación o repercusión al interior de las organizaciones escolares. 
Finalmente, existe un tercer origen que vincula a la escuela con otras variables externas que generan conflictos al interior de estas (Folch, 1995), y que refieren al contexto social, político, económico y cultural en el que se encuentran insertas las instituciones educativas. Debido al rol que desempeñan, las escuelas están altamente permeadas por el contexto (Davies, 2003; Mayer, 2009; Ruiz, 2006), por lo que las transformaciones sociales y los diversos procesos sociopolíticos, como guerras civiles, procesos de segregación étnica, crisis políticas, corrupción, entre otros, tienen efectos al interior del espacio escolar. De esta forma, las distintas dinámicas y procesos sociales repercuten en las organizaciones educativas, manifestándose según las propias lógicas internas del espacio escolar, tal como se ha estudiado recientemente con la desigualdad social (Dupriez, 2010), los niveles de movilidad social o fenómenos como la creciente migración (Pont, Nusche y Moorman, 2009).

\subsection{Dinámicas de los conflictos socioeducativos}

Tal como se ha mostrado, el concepto de conflicto socioeducativo se sustenta fundamentalmente en su fuente de origen. Sin embargo, las formas que adoptan dichos conflictos y sus consecuencias dependen, en gran medida, de las dinámicas y configuraciones que se producen en el propio proceso de conflictividad. En la comprensión de dichas dinámicas en la escuela resulta relevante la distinción realizada por la literatura entre los conflictos cognitivos y los conflictos afectivos (Unline et al., 2003). Mientras los primeros se refieren a desacuerdos y/o disputas en torno a procedimientos, políticas, opiniones y distribución de recursos; los segundos, también catalogados como conflictos identitarios (Wagner, 2005), involucran elementos emocionales, que se asocian con amenazas a la identidad individual o grupal y/o a las normas y valores de las partes involucradas (Unline et al., 2003).

Aunque la distinción es útil en sí misma, lo relevante de esta diferenciación son las trayectorias que sufren ambos tipos de conflictos. Así, mientras los cognitivos tienden a conducir a la creación de contextos donde el desarrollo de opiniones y formas de pensar divergentes son estimuladas (Lima, 2001), los conflictos afectivos 
derivan con mayor facilidad en situaciones de disfunción (Unline et al., 2003), lo que conduce a conflictos destructivos (Deutsch, 1977) o improductivos (De Dreu \& Gelfand, 2008; Stewart, 2015). De este modo, el tránsito de lo cognitivo a lo afectivo aumenta la probabilidad de que el conflicto escale negativamente a una fase en que, de acuerdo con Binaburo y Muñoz (2007), gire más en torno a la reacción de la otra parte, que al problema o asunto que lo originó, lo que frecuentemente genera falta de diálogo y confianza (TschannenMoran, 2014), y un alto grado de personalización, todos aspectos que profundizan la intensidad del conflicto y complejizan su manejo y gestión.

Junto con las trayectorias esperadas, la literatura ha mostrado también de qué manera las consecuencias de los conflictos dependen de la forma en que estos sean abordados y gestionados por las partes involucradas o por la intervención de actores externos (De Dreu \& Gelfand, 2008). Es así que en el caso de las escuelas, se ha señalado que los conflictos tienden a afectar más que a facilitar el funcionamiento organizacional y las relaciones de los actores, principalmente por la falta de herramientas de gestión de conflictos (Lima, 2001). Dada la estructura jerárquica de las escuelas, los directores escolares son vistos como los líderes educativos (Horn y Marfán, 2010), y son los llamados a tener un rol central en el proceso de gestión y manejo de los mismos. Tal como indica Karim (2015), aprender a lidiar constructivamente con los conflictos es una habilidad necesaria para los líderes educacionales, en tanto que estos procesos no manejados pueden conducir a la creación de escuelas disfuncionales (Snodgrass \& Blunt, 2009).

\subsection{Liderazgo directivo y estrategias de manejo de los conflictos socioeducativos}

En los últimos años ha existido un amplio debate a nivel internacional en torno a la importancia del ejercicio de un liderazgo educativo de alta calidad en la introducción de mejoras efectivas en las escuelas y en los resultados académicos de los estudiantes (Bush, 2008; Huber, 2004; Pont et al., 2009). Se ha afirmado incluso que el liderazgo escolar representa la segunda influencia más importante en los 
procesos de aprendizaje después de la docencia (Bush \& Glover, 2012; Leithwood, Day, Sammons, Harris \& Hopkins, 2008; Mitgang, 2012). Sin embargo, y a pesar de su relevancia, el rol del liderazgo directivo en los conflictos socioeducativos no ha sido mayormente tematizado. Aunque estudios realizados en países anglosajones (Bartsch \& Cheurprakobkit, 2002; Coleman \& Fisher-Yoshida, 2004) y América Latina (Weinstein, Cuellar, Hernández y Flessa, 2015) han mostrado la existencia de amplias orientaciones normativas que vinculan a los directivos en la gestión y resolución de los conflictos como una de sus tareas básicas, pocos líderes escolares han contado con la formación necesaria para el manejo constructivo de los conflictos (Anderson, 2005). Adicionalmente, la investigación en la materia es todavía incipiente, concentrándose especialmente en dos aspectos: el rol del director como agente activo en los procesos de conflictividad y la relación entre las formas de manejo de los conflictos y los tipos de liderazgo escolar.

Respecto del rol del directivo como un agente activo en la gestión de los conflictos al interior de las escuelas, Johnson (2003) ha señalado que las dinámicas de los conflictos responden en gran medida a las habilidades de liderazgo del director. En esta línea, se ha indicado que una de las características de los directivos es que, a diferencia de otros actores, tienen amplios márgenes de acción al interior de las organizaciones educativas, lo que les permite ejercer distintos estilos y estrategias de gestión de conflictos. Por lo mismo, el directivo podría entenderse como un actor central en la conflictividad escolar (Boucher, 2013).

Complementariamente, un conjunto de investigaciones ha indicado que el estilo de manejo del conflicto de los directivos constituye un aspecto del estilo de liderazgo educativo ejercido (Boucher, 2013). En tanto que Runde y Flanagan (2010) han definido la competencia en el manejo de los conflictos como la habilidad para desarrollar y usar las habilidades cognitivas, emocionales y de comportamiento con el fin de aumentar los resultados positivos del conflicto, reduciendo con ello la probabilidad de escalamiento negativo. De este modo, obtener resultados positivos implica mejorar la calidad de las relaciones, desarrollando soluciones 
creativas, llegando a acuerdos que permitan enfrentar desafíos y oportunidades similares en el futuro. En esta misma línea, Unline y colaboradores (2003) han sostenido que los líderes deben otorgar una deliberada atención a la cultura organizacional, estructuras y políticas, potenciando un clima organizacional que conduzca a controversias constructivas.

\section{Metodología}

La investigación se desarrolla desde un enfoque cualitativo, buscando levantar información acerca del fenómeno estudiado a partir de las palabras de los individuos y su conducta observable (Taylor y Bogdan, 1987). Por la naturaleza exploratoria y descriptiva del estudio, se utilizó el método de estudio de caso, que se caracteriza por hacer un análisis en profundidad respecto de una serie de unidades muestrales que pueden ser un sujeto o un grupo social, a través de sus manifestaciones y vivencias personales (García Llamas, González y Ballesteros, 2001). Específicamente, el estudio se constituyó como un estudio de casos diferentes (Stake, 1995) que utilizó información generada a partir de las respuestas que se obtienen de forma parcial en casos analizados individualmente (Yin, 1994). De esta forma, se buscó contrastar múltiples fuentes de evidencia "con datos que deben converger en un estilo de triangulación; y, también como resultado, se beneficia del desarrollo previo de proposiciones teóricas que guían la recolección y el análisis de datos" (Yin, 1994, p. 13). Por esta razón, los casos no buscaron generar una representatividad de la realidad social.

El universo muestral del estudio de caso fueron los liceos públicos (municipales) chilenos que imparten solo enseñanza media o secundaria y que se conocen como liceos. Se seleccionaron ocho casos (escuelas) de estudio a través de criterios de heterogeneidad, tanto de las escuelas (ubicación territorial, tipo de formación entregada, rendimiento académico en pruebas estandarizadas y tamaño) como de los directores (distinto sexo, distintos grados de experiencia y al menos con dos años a cargo de la institución). En cada caso, se aplicaron diversas técnicas de recolección de información, siguiendo una aproximación creciente o en espiral, tal como se grafica en la Tabla 1. 
Tabla 1

Técnicas de recolección de información y número de participantes

\begin{tabular}{lcc}
\hline Técnicas de recolección & $\begin{array}{c}\mathrm{N}^{\circ} \text { de } \\
\text { aplicaciones }\end{array}$ & $\begin{array}{c}\mathrm{N}^{\circ} \text { total de } \\
\text { participantes }\end{array}$ \\
\hline Primera entrevista semiestructurada a & 8 & 8 \\
director/a & 8 & 47 \\
Grupo focal docentes & 8 & 54 \\
Grupo focal estudiantes & 8 & 33 \\
Grupo focal apoderados & 8 & 8 \\
Entrevista de incidentes críticos director/a & 40 & 150 \\
\hline Total & & \\
\hline
\end{tabular}

En cada caso, la primera aproximación fue mediante una entrevista semiestructurada (Taylor y Bogdan, 1987) con el director o directora, donde se buscó conocer la percepción de este(a) acerca de los siguientes aspectos:

- las relaciones al interior del establecimiento,

- el liderazgo educativo,

- los niveles de participación y la estructura democrática al interior del liceo,

- los conflictos socioeducativos presentes en él y

- la gestión directiva de los conflictos.

Posteriormente, y como una forma de triangular información, se realizaron grupos focales con docentes, estudiantes y apoderados del establecimiento. Finalmente, se verificó una segunda entrevista con el director, en la que se utilizó la técnica de incidentes críticos (Chell, 2004; Gremler, 2004). Esta técnica posibilita la investigación de hechos identificados por los propios sujetos como significativos (eventos, procesos y/o temas), la manera en que fueron manejados y sus consecuencias. El objetivo es lograr el entendimiento del incidente desde la perspectiva de los propios individuos, tomando en consideración los elementos cognitivos, afectivos y de comportamiento allí contenidos (Chell, 2004). Debido a su naturaleza inductiva, la técnica de incidentes críticos ha sido especialmente útil como método exploratorio para el conocimiento de temas escasamente documentados (Gremler, 2004). En este caso, esta segunda entrevista buscó conocer en profundidad los principales conflictos socioeducativos al interior 
de cada establecimiento en los últimos años y la gestión directiva desarrollada por ellos mismos en estos procesos.

Para el análisis de datos, se utilizó la técnica de análisis del contenido (Krippendorff, 1990; Lincoln \& Denzin, 1994) y se realizó una codificación abierta de las principales categorías planteadas en las preguntas de investigación, destacando y describiendo sus particularidades (Landry, 1998, citado por Gómez Mendoza, 2000).

\section{Resultados}

Los resultados se organizan en tres apartados, relacionados con los objetivos de investigación. El primero describe los principales conflictos socioeducativos encontrados, dando cuenta de los actores involucrados y los niveles en los que estos se han desarrollado. La segunda parte se focaliza en describir las dinámicas y formas en que se desarrolla el conflicto, mientras que el tercer apartado detalla las estrategias de gestión y manejo de los conflictos utilizados por los directivos.

\subsection{Frecuencia y características de los conflictos socioeducativos}

En general, es posible visualizar la existencia de múltiples temáticas, demandas, actores involucrados y causas de los conflictos educativos, que involucran disputas de poder por liderazgos, procesos de agobio laboral, conflictos originados por movilizaciones estudiantiles y problemas de adaptación de nuevos actores a la dinámica escolar, entre otros aspectos. Sin embargo, y a pesar de esta heterogeneidad, se pueden apreciar algunos patrones comunes que vale la pena analizar.

Por una parte, se observa que las fuentes del conflicto tienden a variar según los actores involucrados. Así, cuando los conflictos involucran a los estudiantes como actores centrales, estos tienen mayoritariamente un origen contextual a la escuela, es decir, tienden a asociarse con el contexto social, económico y cultural del que provienen las familias de los estudiantes. Por el 
contrario, cuando los conflictos involucran a adultos (generalmente profesores y directivos) en general estos tienen como fuente de origen problemáticas de la micropolítica y, en segunda instancia, del campo educativo o macropolítica escolar.

En relación con los conflictos que involucran a los estudiantes, existen fenómenos como las peleas y riñas entre ellos, los conflictos asociados con la indisciplina escolar y controversias producidas por casos de bullying y ciberbullyng, que pueden ser interpretados como distintas expresiones de la violencia escolar y están presentes en todos los establecimientos, lo que concuerda con la literatura existente en el tema (Córdoba-Alcaide et al., 2016; D’Angelo y Fernández, 2011; Magendzo \& Toledo, 2012). Sin embargo, también existen factores contextuales que son fuente de conflictos al interior de las escuelas y que no han sido relevados como elementos fundamentales anteriormente. Al respecto, aparece como especialmente importante la problemática del consumo y tráfico de drogas entre estudiantes, la que se encuentra presente en todos los establecimientos estudiados y que concita una gran preocupación entre los actores escolares, tal como se desprende de las siguiente cita:

El mayor problema que tenemos en estos momentos, es cómo se está introduciendo la drogadicción, ese es el mayor tema, lo que más nos preocupa. Nosotros no tenemos grandes problemas de indisciplina, pero sí de drogadicción. Ya se está traficando al interior del liceo (...) Primero fumaban, luego la pipa, luego una lapicera que se la ponen la boca y están consumiendo. Hemos cerrado todos los lugares donde pueden consumir y es terrible. Hay algunos que llegan drogados de su casa (...) Los profesores tienen una mirada bastante crítica respecto al tema, incluso han señalado que para ellos es riesgoso. Porque si llegan alumnos en ese estado, de consumo, puede aflorar la agresividad. Los profesores se sienten inseguros teniendo alumnos drogados dentro de la sala, pueden tener cualquier reacción con otro alumno o con el mismo profesor. Es un hecho recurrente en los consejos de profesores que aparezca el tema, y siempre nosotros con la misma explicación, la que tenemos, los elementos de juicio que nos otorga la ley (Director, liceo 1). 
Como se puede observar, la existencia y consumo de drogas es visualizado como un problema que no solo genera dificultades de aprendizaje dentro de los estudiantes, sino que también impacta en el clima escolar y la organización funcional del establecimiento, siendo, por lo mismo, una fuente de múltiples y constantes conflictos.

En esta misma línea, otra fuente contextual de conflictos focalizada en los estudiantes dice relación con los ambientes donde estos se desenvuelven fuera de la escuela. Al respecto, los distintos actores educativos son especialmente críticos respecto de las pautas culturales y sociales existentes en los hogares, lo que genera una fuente permanente de tensión entre profesores y apoderados. Así, son frecuentes en los establecimientos conflictos provocados por el bajo compromiso y ausencia de las familias en la educación de sus hijos o los patrones culturales entregados por el hogar, como se refleja en las siguientes citas:

Yo le digo (al apoderado), "mire su hijo tiene esta problemática. Bueno y usted po' (sic), péguele Ud". Yo le digo "yo no le puedo pegar, veamos la estrategia"... "No, es que ahora no puedo, estoy ocupada, yo tengo que ir a trabajar" (Docente, liceo 8).

Estos niños no tienen cultura de llegar a la sala, de saludar, de sentarse, sacar el cuaderno, escribir... eso les falta, que tengan un mínimo de cultura del tema también del respeto por la autoridad (Docente, liceo 2).

A diferencia de lo que ocurre con los conflictos originados por el microtráfico o la drogadicción, en estos casos los conflictos son visualizados más como tensiones que como disputas, quedando relegados muchas veces al plano de la conflictividad latente o potencial (Wagner, 2005).

Además de los conflictos que involucran a estudiantes, aquellos que competen a los adultos también están presentes en parte importante de los casos, siendo especialmente relevantes aquellos que implican a profesores y directivos. Como se indicó anteriormente, en su mayoría, estos conflictos tienen su origen 
en la micropolítica y en la macropolítica de los establecimientos escolares.

Una fuente común de conflicto entre docentes y también entre docentes y directivos son los procesos de movilización social de los profesores ${ }^{8}$, los que adquieren especial importancia durante los paros docentes. En estos casos, tanto docentes como directores reconocen las movilizaciones como una fuente de conflicto por múltiples motivos. Es así que, en algunos casos, el conflicto se produce porque la movilización genera transformaciones en la distribución de poder al interior de la escuela, mientras que en otros los conflictos son parte de las consecuencias prácticas de los procesos de movilización:

(...) Asoman caudillismos, otros liderazgos, el que era representante de los profesores renuncia, no quiere nada más porque fue sobrepasado. Lo criticaron porque en un momento era la tendencia, después la otra. "¿Cómo si soy de este gobierno, cómo voy a estar siendo partícipe de una huelga?", se forma todo un torbellino y se arma conflicto entre los docentes (Director, liceo 1).

Ahí es donde empieza el tema, cómo recuperamos [las clases perdidas por el paro] y recuperar significa venir el sábado... los que van a paro nunca aparecen los sábados, entonces ¿dónde está la consecuencia? Entonces ahí es donde chocamos un poco, por lo obvio, si yo voy a paro tengo que ser consciente que voy a tener que recuperar, y esa conciencia no existe en algunos (Director, liceo 2).

Otra causa recurrente de conflicto se relaciona con las condiciones laborales de los docentes. Al contrario de lo que pudiera pensarse, los principales conflictos a este respecto no se producen por asignaciones o bonos ni por posibles aumentos de remuneraciones, sino que se originan principalmente por la carga

8 Desde la perspectiva del estudio del campo educativo y su relación con el campo político, algunas investigaciones han destacado la importancia de este tipo de conflictos, aunque siempre se han focalizado en su efecto en la esfera extraescolar (Leiras, 2007; Weinstein, 2007). 
laboral ${ }^{9}$ a la que se ven enfrentados los docentes y a los crecientes niveles de "agobio laboral" existentes. De este modo, es frecuente que los profesores aludan a la sobrecarga laboral como un problema, lo que para algunos directores provoca una permanente tensión que muchas veces deviene en abiertos conflictos.

Lo que pasa es que los profesores nos sentimos acosados, con un agobio laboral. Se consiguió un personero (sic) del Ministerio [de Educación] que nos diera una charla sobre este asunto. Y ahí nos llamó la atención que el caballero dijo claramente que los directivos estaban al servicio de los profesores y no al revés. Y nosotros seguimos estando al servicio de los directivos, tenemos que entregarles prácticamente todas las cosas listas (Docente, liceo 3).

Finalmente, los conflictos producidos por diferencias en las evaluaciones del trabajo y las disputas de poder entre liderazgos docentes y directivos son también fuentes de conflictos identificadas. En todos estos casos, lo fundamental es que los actores involucrados analizan las problemáticas como fenómenos que transforman y reorganizan las relaciones de poder al interior de la escuela, tal como se observa en la siguiente cita:

... [un docente] él tiene una visión bastante crítica del colegio y las veces que va lo único que hace es ir a cuestionar y a criticar lo que se está haciendo (...) los consejos de profesores se transformaban en un diálogo entre él y yo, él hablaba en nombre de todos los colegas, que esto sí, que esto no (...) Él resintió harto el haber perdido protagonismo y liderazgo entre sus pares, entonces se generó un conflicto entre él y yo (Director, liceo 4).

En síntesis, la frecuencia y características de los conflictos analizados sobrepasa con creces a las relaciones que se producen entre determinados actores o a las causas que se originan solo al interior o al exterior de la escuela, pudiendo entenderse como un

$9 \quad$ La cantidad de horas laborales de los docentes en Chile (1.103 horas anuales) supera por mucho al promedio de los países que componen la OECD (694 horas anuales). Asimismo, el tiempo dedicado a la preparación de clases (5,8 horas) se encuentra por debajo del promedio de los países OECD $(7,1)$ (OECD, 2014). 
fenómeno complejo y multidimensional, que involucra múltiples acciones y que se gatilla por procesos diversos.

\subsection{Dinámicas en los conflictos socioeducativos}

Además de la fuente de los conflictos, es interesante analizar las trayectorias y dinámicas que determinan cuál es el resultado o las consecuencias que tienen estos fenómenos. En este sentido, los resultados del estudio muestran especialmente tres características diferenciadoras de esta dinámica en los liceos públicos chilenos: su tránsito desde conflictos cognitivos a conflictos afectivos; los altos niveles de personalización que adquieren los conflictos; y las consecuencias improductivas que producen estos conflictos.

Respecto del primer aspecto, se pudo constatar que una característica común de los conflictos socioeducativos analizados fue que muchos comenzaban entendiéndose como conflictos cognitivos, pero con el paso del tiempo devenían en conflictos afectivos. De esta forma, los conflictos tendían a situarse en el plano de las relaciones interpersonales y afectivas, aun cuando su inicio u origen estuviera relacionado con aspectos políticos, sociales o ideológicos del quehacer escolar. Un ejemplo de esta transición es la situación que relata un docente respecto del conflicto entre el director del establecimiento y otro docente, en el marco de las movilizaciones gremiales:

El año pasado cuando tuvimos los problemas del paro, hubo fuertes encontrones [entre el director y él] que era líder de la parte gremial del Colegio de Profesores. Cosas que no debieron haber pasado nunca en un Consejo de Profesores, desde mi perspectiva, como decirse cosas personales e hirientes. (...) si bien yo no lo conocía de antes [al director], ahí me empezó a quedar una impresión. Yo hago mi pega y lo respeto, trato de ser funcional en ese aspecto. Pero yo ya quedé con cierta aprehensión y tengo un trato bien limitado con él (Docente, liceo 1).

Así, el tránsito de lo cognitivo a lo afectivo produce una personalización del conflicto. Esto implica que estos suelen 
describirse como conflictos entre personas más que por situaciones, lo que implica que la disputa tiende a centrarse en las características personales de los actores. Un ejemplo de esta dinámica puede observarse en los conflictos generados por los resultados de una evaluación docente, que conducen a animadversiones que conllevan descalificaciones personales, tal como relata una directora:

Entonces yo llamo al profesor a esta retroalimentación [de una evaluación en aula] y le digo todas estas cosas y reaccionó súper mal, y en vez de decir algo autocrítico, se defendió todo el tiempo, entonces cuando ya se sintió muy cuestionado, me empezó a mí a cuestionar en mi rol de directora: "usted que entró a este liceo, seguramente tiene algún conocido", cosas así. Y la verdad es que la conversación se tornó súper absurda, se fue, y yo me quedé sin entender nada, porque además era el primer año [en su rol como directora] (...) luego con algunos cursos, de manera insistente hablaba mal de mí, les decía que mi nombramiento no era oficial porque no había sido elegida por la alta dirección pública (Directora, liceo 8).

Al tornarse personales y escalar negativamente, los conflictos dejan de estar asociados al rol de los actores en la escuela y entran en el terreno de las relaciones interpersonales y de las emociones. Esto hace que se olvide lo que originó el problema, y el conflicto vaya transformándose en una suma de nuevos eventos y diferencias de tipo personales. Por ejemplo, en uno de los liceos donde el consumo y tráfico de drogas es una de las principales problemáticas, los docentes y paradocentes decían sentir temor de la reacción que podrían tener los alumnos frente a las acciones que ellos tomaran al sorprenderlos:

Yo te pongo la siguiente situación: "profesor Mario, hay cuatro alumnos en malas condiciones". El profesor Mario hace la pega que tiene que hacer, llama al inspector y hace que lo retiren de la sala. Esos mismos cuatro al otro día con el auto del profesor Mario afuera... uno corre el gran riesgo de que ellos pasen, un rayón, un vidrio, cualquier cosa (Docente, liceo 1). 
Los inspectores, cuidadores de patio, no hacen nada. Hablan de que se va a controlar más, pero al final no hacen nada, es como "le tenemos miedo a los alumnos"; no se ponen bien los pantalones como para decirte esto está mal y esto está bien... (Estudiante, liceo 1).

Esta reflexión puede iluminar la tercera característica de la dinámica de los conflictos encontrados: su carácter improductivo. Así, cuando se entra en el terreno de lo afectivo y en la personalización, los conflictos pueden volverse improductivos o pueden escalar negativamente (Glasl, 2004). Siguiendo con el ejemplo anterior, el temor hacia la respuesta de los alumnos hace que no siempre se tomen las medidas necesarias para solucionar el conflicto y finalmente este se estanque o escale, lo que genera un círculo vicioso de contradicciones y confusiones. Eso explica que los directivos y docentes de este liceo se sientan sin herramientas para afrontar el problema efectivamente, tal como se desprende de la siguiente cita:

Creo interpretar a todos los directores de mi país, nosotros en este sentido lo único que podemos hacer es trabajar medidas preventivas no más, y cuando corresponda, hacer las denuncias (Director, liceo 1).

De esta manera, la dinámica de conflictividad que se desarrolla en las escuelas potencia procesos de personalización y afectividad que hacen que, en muchos casos, los conflictos sean visualizados como procesos negativos que no contribuyen al desarrollo de las instituciones escolares.

\subsection{Estrategias de gestión directiva de los conflictos socioeducativos}

Tal como ha sido mencionado, la gestión de los conflictos al interior de los establecimientos escolares resulta ser un aspecto importante en el ejercicio del liderazgo escolar. En el estudio de caso presentado, es posible visualizar tres hallazgos principales en relación con este tópico. En primer lugar, las formas de manejar y gestionar los conflictos dependen en gran medida del tipo de actor involucrado: 

EXPLORATORIO EN LICEOS PÚBLICOS CHILENOS - C. Villalobos, J. Peña, E. Aguirre y M. Guerrero

si el conflicto es entre estudiantes, para los que existen reglamentos y protocolos de acción ${ }^{10}$, se procede de una forma muy distinta a cuando los conflictos involucran adultos (docentes y directores), para los que, en general, no existen conductos regulares a seguir. El segundo hallazgo refiere a que entre los casos analizados, las estrategias que se utilizan son tan diversas como los propios conflictos y, por lo tanto, las respuestas son situacionales más que estructurales. Finalmente, los resultados muestran que, mientras los conflictos relacionados con los estudiantes buscan ser resueltos, los conflictos relativos a adultos tienden a ser invisibilizados o evitados.

Respecto del primer aspecto, se pudo constatar que para diversos conflictos que involucran a estudiantes, especialmente para los casos más complejos (aquellos relacionados con situaciones de abuso sexual o consumo y tráfico de drogas y las problemáticas que se derivan de estas situaciones) los establecimientos escolares cuentan con protocolos de acción ${ }^{11}$, generados a partir de las orientaciones ministeriales o establecidos por las propias escuelas, por lo que las estrategias de manejo o gestión del conflicto se limitan al cumplimiento de dichos procedimientos por parte de los directivos, dando cuenta de una visión que podríamos llamar protocolar.

10 Durante la última década, las autoridades ministeriales han desarrollado un conjunto de instrumentos de política que buscan orientar el quehacer de la escuela sobre esta materia. Así, en el 2002 surge la primera política a nivel nacional respecto de la convivencia escolar. Posteriormente, en 2008, el Ministerio de Educación establece el sitio www.convivenciaescolar.cl para orientar a los establecimientos en esta área. Años más tarde, en 2011, se promulga la Ley N 20.536 Sobre Violencia Escolar, en donde se establecen los marcos de lo que se entiende por convivencia escolar. Esta ley, además, establece el nombramiento de un encargado de convivencia escolar en todos los establecimientos escolares del país, entrega nuevas tareas a los Consejos Escolares y obliga a los colegios a contar con un reglamento interno que regule las relaciones entre el establecimiento y los distintos actores de la comunidad escolar (Ministerio de Educación de Chile, Mineduc, 2015; 2016).

11 En general, estos protocolos están desarrollados en el marco de la legislación existente. Así, por ejemplo, la Ley de Violencia Escolar tipifica el acoso escolar, entendido "como toda acción u omisión constitutiva de agresión y hostigamiento reiterado, realizada fuera o dentro del establecimiento educacional por estudiantes, que en forma individual o colectiva, atente en contra de otro estudiante". Adicionalmente, se establece como de "especial gravedad cualquier tipo de violencia física o psicológica, cometida por cualquier medio en contra de un estudiante integrante de la comunidad educativa, realizada por quien detente una posición de autoridad, sea director, profesor, asistente de la educación y otro". Estas normativas han sido complementadas o reforzadas por otras normativas, como la Ley No 20.069 de No Discriminación (2012) o la Ley de Inclusión No 20.845 (2015) (Mineduc, 2015). 
Bueno, [yo actúo] aplicando la Ley 20.000 no más, no hay otra forma, la ley es clarita, si yo no procedo en lo que dice la ley, soy un cómplice y yo no voy a ser cómplice. Entonces haré lo que la ley dice. Yo encuentro a los alumnos portando [droga], yo los denuncio. En ese aspecto soy intransigente (Director, liceo 2).

Esto ya está normado de la Fiscalía, o sea desde el Ministerio de Justicia. Nosotros hemos recibido estas orientaciones en, por los menos, tres consejos, o sea, la cosa ahora está así y no es que tú quieras o no quieras, tú debes y está todo muy clarito (Docente, liceo 6).

Ahora bien, pese a aplicar medidas protocolares frente a conflictos de alta complejidad, en algunos casos más graves como los generados por problemáticas de drogas, los directores declaran cierta incapacidad en sus posibilidades de acción, lo que se condice con la percepción de insuficiencia frente a estos temas que tienen las comunidades escolares.

En contraste, cuando los conflictos entre estudiantes se producen por disputas menores, los manuales de convivencia escolar entregan distintas alternativas, como la mediación, la negociación o el arbitraje, dando más margen de acción al directivo. Frente a esta situación, algunos directores tienden a mantener una actitud protocolar, ateniéndose a los marcos de regulación existente. Así, por ejemplo, uno de los liceos del estudio cuenta con un Comité de Resolución de Conflictos Escolares, que cumple la labor de analizar y resolver sanciones en relación con una falta cometida, el cual está constituido por el director, inspectores generales, profesor jefe y representante del Centro de Alumnos. En contraste, en otros casos los actores declaran actuar más libremente, tal como describe un docente de un establecimiento:

Ahora hay protocolo para todo, de convivencia escolar (...) pero muchas veces los conflictos se arreglan conversando. Muchas veces Juan con Pedro pelean a combos. Si yo pescara el protocolo que ahora me exigen, me demoraría siglos en solucionar el conflicto (Docente, liceo 6). 
En contraste con estos casos, cuando los conflictos involucran a docentes y directivos no existen mayores normativas que permitan definir estrategias de manejo de conflictos, y, por lo tanto, las estrategias adoptadas por los directivos dependen en gran medida del criterio personal y, por ello, son altamente heterogéneas. Asimismo, en estos casos, los conflictos son narrados por los directores como hechos aislados, siendo incluso vistos como hechos naturales considerando el cargo que desempeñan. En esta línea es que uno de los directores se refiere a las tensiones y distancia que tenía con algunos docentes:

No es generalizada tampoco, yo creo que la gran mayoría de los funcionarios apoya la labor directiva del equipo, pero como todas las cosas en la vida, hay oposición, gente que no se siente identificada [Esto] se da en todas partes, por la impronta que tiene el director (Director, liceo caso 1).

(...) Cuando gusten de conversar conmigo, pueden venir, no tengan ningún temor, díganme sus inquietudes, pero no lo hacen. Entonces por ejemplo, en la sala de profesores, si yo entro a la sala de profesores, están todos ahí conversando, tomándose su té, pero entra el director, subdirector o inspector general, un silencio absoluto, se cambian los temas, ya no se conversa de lo que se estaba conversando (Director, liceo 1).

De esta manera, los conflictos entre adultos son, en gran medida, invisibilizados y percibidos por los directivos como procesos de tensión natural, con la excepción de aquellos casos donde la situación es grave y los involucra a ellos más directamente. Un ejemplo de esta situación se puede apreciar en la siguiente cita, donde una directora señala que su estrategia es no involucrarse en los problemas entre sus docentes y continuar con los mecanismos habituales de trabajo:

Yo de verdad que mucho no le he puesto [cuando ha habido conflictos entre docentes]. Yo sigo trabajando como corresponde, dando las órdenes que corresponden, e indicándole a la gente cuál es su trabajo y listo, yo no puedo empezar a solucionar todos los problemas entre ellos (Directora, liceo 3). 
Un segundo hallazgo está relacionado con el margen de acción y las estrategias de gestión adoptadas, donde se puede constatar que, en general, existe una multiplicidad de acciones implementadas que dependen y varían según las distintas necesidades, contextos y habilidades personales. Esto implica que las estrategias de manejo de los conflictos son bastante situacionales, y no responden a un patrón claro relacionado con los estilos de gestión o alguna característica particular del directivo. Así, por ejemplo respecto de los conflictos producidos por problemas de drogas, las estrategias son múltiples: en algunos casos, se aplican los protocolos jurídicos establecidos, en otros se implementan iniciativas de apoyo (a través de procesos de intervención psicosocial o implementación de actividades extraprogramáticas), mientras que en otros momentos se establecen redes con instituciones externas, ya sea para generar programas de prevención como para realizar procesos de derivación. Finalmente, algunos directivos optan por estrategias más coercitivas, como el uso de cámaras dentro del liceo o la revisión de mochilas. Así, las estrategias tienden a ser adoptadas in situ por los directivos, a partir de la evidencia existente (y en algunos casos, apoyadas en la opinión de otros miembros de la comunidad educativa). Esto deriva en la dificultad de establecer relaciones entre el estilo de liderazgo que declaran ejercer y las estrategias que adoptan frente a los conflictos.

Finalmente, los resultados del estudio de caso mostraron que las estrategias de los directores en los conflictos que involucran a estudiantes responden a un enfoque de resolución, en el que se busca que este sea erradicado más que manejado, mientras que en el caso de los conflictos que involucran a adultos es común que para los directores estos no necesariamente requieran de acciones concretas que los gestionen, por lo que tienden a ser evitados. En este sentido es que puede entenderse la siguiente cita, mencionada por un director al relatar el conflicto con un docente:

Nosotros lo conversamos [con el equipo directivo] y buscábamos estrategias, Y una de las estrategias fue que yo no conversaba más con él. Cuando había que decirle algo a él, no lo iba a hacer más yo, lo iba a hacer el inspector general. O cuando había que dar una información que iba a generar conflictos en él, la daba el inspector. 
Otra estrategia: todo por escrito. Entonces él me mandaba una nota y yo le respondía (Director, liceo 4).

Esta tendencia a no enfrentar los conflictos entre adultos puede estar vinculada con que la función directiva se asocia más a aspectos administrativos y al cumplimiento de sus funciones de líder pedagógico que a la construcción y promoción de climas laborales óptimos, en los que los conflictos se gestionen constructivamente.

\section{Conclusiones}

Cuatro conclusiones principales pueden establecerse a partir del estudio realizado. La primera de ellas refiere a la multiplicidad de conflictos socioeducativos presentes en los establecimientos escolares. Como se pudo observar, las escuelas presentan conflictos que tienen su origen tanto en temas que surgen al interior de ellas (micropolítica escolar) como en otros provenientes del exterior (macropolítica y/o contexto). Este hallazgo, aunque pueda parecer lógico, plantea la necesidad de ampliar el foco y la mirada de la conflictividad escolar (tradicionalmente puesto en la violencia entre estudiantes) incorporando en el análisis los múltiples y complejos actores y factores que se relacionan con los conflictos en las organizaciones escolares.

Una segunda conclusión, relacionada con la anterior, refiere a la centralidad de los actores involucrados en el desarrollo y gestión de los conflictos. Tanto en el tipo de conflictos como en las estrategias de gestión parece haber una diferencia fundamental en los casos en los que los conflictos involucran a estudiantes, en contraste con los casos que involucran a adultos, lo que podría producirse por el rol diferenciado que se entrega a cada actor en el espacio escolar, visualizando al niño o niña como un actor más dúctil y con menos capacidad de negociación.

Un tercer aspecto pesquisado se refiere a la especificidad de las dinámicas de los conflictos en las escuelas. Los conflictos socioeducativos desarrollados en escuelas chilenas tienden a transitar, con mayor frecuencia, desde lo cognitivo a lo afectivo, lo que hace 
más probable que escalen negativamente y se vuelvan conflictos improductivos. En este escenario, estos dejan de estar asociados al rol de los actores en la escuela y se circunscriben más bien al terreno de las relaciones interpersonales y las emociones, lo que se constituye como un hallazgo central para la construcción de futuras políticas públicas en la materia.

Por último, un cuarto hallazgo se vincula con las múltiples estrategias desplegadas por los directores al momento de abordar los conflictos socioeducativos. Las estrategias son situacionales y tan diversas como los propios conflictos, siendo difícil establecer patrones comunes que permitan identificar tipos de estrategias o establecer posibles vínculos entre ellas y el estilo de liderazgo directivo ejercido. Así, la pluralidad de estrategias y el actuar de acuerdo con la contingencia podrían ser reflejo de la escasa formación que los directivos reciben respecto de este tema, lo que interpela a la política gubernamental de formación directiva.

Vistos en conjunto, estos hallazgos son una primera aproximación exploratoria al estudio de los conflictos y al rol de los directivos en Chile y América Latina, y se necesita nueva evidencia e investigación que permita complementar o contrastar estos resultados. Es posible visualizar a lo menos dos posibles ampliaciones en esta línea. Por una parte, el estudio se enfocó en un solo tipo de escuela: los liceos municipales que imparten enseñanza desde el nivel secundario, dejando de lado los establecimientos particulares y particulares subvencionados, como también a aquellos que inician sus clases en los cursos inferiores. Conocer en forma cualitativa y/o cuantitativa la dinámica de desarrollo de los conflictos en estos otros espacios y las estrategias de negociación desarrolladas por estos directivos permitirá ampliar el campo de estudio a otras realidades. Al mismo tiempo, es necesario reconocer la especificidad del caso chileno (Bellei, 2015; Villalobos, 2016) como un sistema basado en la lógica del mercado, la competencia escolar y la privatización, lo que abre la interrogante sobre el impacto de estas políticas en nuestro tema de investigación. En esta línea, es necesario reconocer que tanto los conflictos como las estrategias de gestión de los mismos no son extrapolables a 
otras realidades, por lo que se requieren estudios comparativos para visualizar patrones comunes a otros sistemas.

El desarrollo y profundización de estas investigaciones permitirá ampliar el campo de discusión acerca de la conflictividad y el liderazgo directivo en la región, permitiendo adentrarse en la búsqueda de tendencias que permitan identificar tipologías de estrategias, así como la comprensión de las lógicas de acción detrás de cada una de las estrategias desplegadas. Esto permitirá realizar un estudio de los conflictos y el rol de los docentes considerando factores como el peso del mercado, las políticas educacionales implementadas y las propias demandas de los actores al interior de las comunidades escolares, reconociendo su relación con la concepción que los directivos tienen respecto de los conflictos al interior de sus escuelas y en las estrategias que emplean para afrontarlos, aspectos fundamentales para entender de mejor forma la dinámica contemporánea de la conflictividad escolar.

\section{Referencias}

Anderson, M. (2005). Conflict management and the prospective principal. Academic Exchange Quarterly, 9(2), 146-150.

Ball, S. (1989). La micropolítica de la escuela. Barcelona: Ediciones Paidós Ibérica.

Bartsch, R. \& Cheurprakobkit, S. (2002). School problems and learning about crime and justice systems. Principals' views. Educational Studies, 28(3), 275-285.

https://doi.org/10.1080/0305569022000003726

Bellei, C. (2015). El gran experimento. Mercado y privatización de la educación chilena. Santiago de Chile: LOM.

Binaburo, J. A. y Muñoz, B. (2007). Educar desde el conflicto. Guía para la mediación escolar. Barcelona: Grupo Planeta (GBS).

Boucher, M. (2013). The relationship of principal conflict management style and school climate (Doctoral dissertation), University of South Caroline, Columbia, SC, USA. Recuperado de http://scholarcommons.sc.edu/etd/948

Bourdieu, P. (1994). Capital cultural, escuela y espacio social. Buenos Aires: Siglo Veintiuno Ediciones. 
Bush, T. (2008). Leadership and management development in education. London: Sage Publications.

Bush, T. \& Glover, D. (2012). Distributed leadership in action: Leading highperforming leadership teams in English schools. School leadership y management, 32(1), 21-36.

https://doi.org/10.1080/13632434.2011.642354

Chell, E. (2004). Critical incident technique. En C. Cassell \& G. Symon (Eds.), Essential guide to qualitative methods in organizational research (pp. 4560). London: Sage Publications.

Centro de Estudios de Políticas y Prácticas en Educación, CEPPE. (2013). Learning standards, teaching standards and standards for school principals: A comparative study. Paris: OECD.

Coleman, P. \& Fisher-Yoshida, B. (2004). Conflict resolution across the lifespan: The work of the ICCCR. Theory into practice, 43(1), 31-38. https://doi.org/10.1353/tip.2004.0003

Córdoba-Alcaide, F., Ortega-Ruiz, R. y Nail-Kröyer, O. (2016) Gestión de la convivencia y afrontamiento de la conflictividad escolar y el bullying. Santiago: RIL Editores.

D’Angelo, L. y Fernández, D. (2011) Clima, conflictos y violencia en escuela. México D.F: Fondo de las Naciones Unidas para la Infancia (Unicef) y Facultad Latinoamericana de Ciencias Sociales (Flacso).

Davies, L. (2003). Education and conflict: Complexity and chaos. New York: Routledge.

Day, C., Sammons, P., Hopkins, D., Leithwood, K., \& Kington, A. (2008). Research into the impact of school leadership on pupil outcomes: Policy and research contexts. School Leadership and Management, 28(1), 5-25. https://doi.org/10.1080/13632430701800045

De Dreu, C. \& Gelfand, M (2008). The psychology of conflict management in organizations. New York: Lawrence Erlbaum Associates.

Deutsch, M. (1977). The resolution of conflict: Constructive and destructive processes. New Haven, CT: Yale University Press.

Dupriez, V. (2010). Methods of grouping learners at school. Paris: Unesco.

Folch, T. (1995). Los conflictos organizativos, factor clave para el desarrollo organizativo de los centros educativos. Educar, 19, 61-76. Recuperado de http://educar.uab.cat/article/download/v19-tomas/413

García Llamas, J. L., González, M. A. y Ballesteros, B. (2001). Introducción a la investigación en educación. Madrid: UNED. 
Glasl, F. (2004). Konfliktmanagement. Ein Handbuch für Führungskräfte, Beraterinnen und Berater. Aktualisierte Auflage. Berlin: Hauptverlag.

Gómez-Mendoza, M. (2000). Análisis de contenido cualitativo y cuantitativo: definición, clasificación y metodología. Revista de Ciencias Humanas, 20, 103-113. Recuperado de http://www.mediafire.com/?4glu9ngyns9xhq4

Gremler, D. (2004). The critical incident technique in service research. Journal of Service Research, 7(1), 65-89. https://doi.org/10.1177/1094670504266138

Gross, S. \& Shapiro, J. (2013). The New DEEL (Democratic Ethical Educational Leadersip) and the Work of Reclaiming a Progressive Alternative in Educational Leadership from Pre-K-20. The International Journal of Progressive Education. 9(3) 49-69.

Gunter, H. (2016). An intellectual history of school leadership practice and research. London: Bloomsbury Publishing.

Handford, V. \& Leithwood, K. (2013). Why teachers trust school leaders. Journal of Educational Administration, 51(2), 194-212. https://doi.org/10.1108/09578231311304706

Horn, A. y Marfán, J. (2010). Relación entre liderazgo educativo y desempeño escolar: revisión de la investigación en Chile. Psicoperspectivas. Individuo y Sociedad, 9(2), 82-104. https://doi.org/10.5027/psicoperspectivas-vol9-issue2-fulltext-116

Huber, S. G. (2004). School leadership and leadership development-adjusting leadership theories and development programs to value and the core purpose of school. Journal of Educational Administration, 6(42), 669684. https://doi.org/10.1108/09578230410563665

Jares, X. (1997). El lugar del conflicto en la organización escolar. Revista Iberoamericana de Educación, 15(1), 53-74. Recuperado de http://rieoei.org/oeivirt/rie15a02.htm

Johnson, P. (2003). Conflict and the school leader: Expert or novice. Journal of Research for Educational Leaders, 1(3), 28-45. Recuperado de https://www2.education.uiowa.edu/archives/jrel/spring03/ Johnson_0204.pdf

Karim, D. (2015). Managing conflict by school leadership: A case study of a school from Gilgit-Biltistan. International Journal of Innovation and Research in Educational Sciences, 2(5), 340-343. Recuperado de http://ecommons.aku.edu/pakistan_ied_pdcn/45/ 
Krippendorff, K. (1990). Metodología de análisis de contenido. Teoría y práctica. Barcelona: Paidós Comunicación.

Leithwood, K., Day, C., Sammons, P., Harris, A., \& Hopkins, D. (Eds.) (2008). Successful school leadership. What it is and how it influences pupil learning. Nottingham: National College for School Leadership.

Leithwood, K., Seashore, K., Anderson, S., \& Wahlstrom, K. (2004). Review of research: How leadership influences student learning. New York: The Wallace Foundation.

Leithwood, K., Tomlinson, D., \& Genge, M. (1996). Transformational school leadership. En K. Leithwood, J. Chapman, D. Corson, P. Hallinger, $\&$ A. Hart (Eds.), International handbook of educational leadership and administration (pp. 785-840). Boston: Kluwer Academic Publishers.

Leiras, M. (2007). Estudio de caso: la relación entre el Ministerio de Educación y el colegio de profesores de Chile, 1990-2006. Cooperación e institucionalización en la determinación de los salarios y las condiciones de trabajo docente. Buenos Aires: Centro de Estudios en Políticas Públicas, Banco Mundial.

Lima, J. (2001). Forgetting about friendship: Using conflict in teacher communities as a catalyst for school change. Journal of Educational Change, 2(2), 97-122. Recuperado de https://link.springer.com/article/10.1023/A:1017509325276

Lincoln, Y. \& Denzin, N. (1994). The fifth moment. En Denzin, N \& Lincoln, Y. (Eds.). Handbook of qualitative research (Vol. 1) (pp. 575-586). London: Sage Publications.

Loyo, A. (2001). Los sindicatos docentes en América Latina: entre la lógica laboral y la lógica profesional. Revista Iberoamericana de Educación, 25(1), 65-81. Recuperado de http://rieoei.org/rie25a03.PDF

Madriaza, P. y García, M. (2004). Clamores juveniles y su relación con la violencia escolar: conflicto y aspiración por recrear un orden social. Persona y Sociedad, 18(1), 31-50.

Magendzo, A. \& Toledo, M. (2012). Bullying: An analysis from the perspective of human rights, target group and interventions. The International Journal of Children's Rights, 21(1), 1-13. https://doi.org/10.1163/157181812x637136

Mayer, L. (2009). Escuela, integración y conflicto. Notas para entender las tensiones en el aula. Educación, Lenguaje y Sociedad, 6(6), 85-112.

Ministerio de Educación de Chile, Mineduc. (2015). Politica nacional de convivencia escolar 2015-2018. Santiago de Chile: Autor. 
Ministerio de Educación de Chile, Mineduc. (2016). Orientaciones para la revisión de los reglamentos de convivencia escolar. Santiago de Chile: Autor.

Mitgang, L. (2012). The making of the principal: Five lessons in leadership training. perspective. New York: Wallace Foundation.

Msila, V. (2012). Conflict management and school leadership. Journal of Communication, 3(1), 25-34. Recuperado de https://www.researchgate.net/publication/233868808_Conflict_ Management_and_School_Leadership

Organisation for Economic Co-operation and Development, OECD. (2014). Education at a Glance 2014: OECD Indicators. Paris: OECD Publishing.

Pont, B., Nusche, D., y Moorman, H. (2009). Mejorar el liderazgo escolar, Volumen 1: política y práctica. Paris: OECD Publishing.

Quezada, M., Navarro, J., y Mora, J. (2005). La violencia escolar. Revista Mexicana de Investigación Educativa, 10(27), 1027-1045. Recuperado de http://www.redalyc.org/pdf/140/14002704.pdf

Román, M. \& Murillo, J. (2011). Latin America: School bullying and academic achievement. CEPAL Review, 104(1), 37-53. Recuperado de http://www.cepal.org/publicaciones/xml/2/45332/ rvil04romanmurillo.pdf

Ruiz, L. (2006). La escuela: territorio en la frontera. Tipología de conflictos escolares según estudio comparado en Bogotá, Cali y Medellín. Recuperado de http://bibliotecavirtual.clacso.org.ar/ar/libros/colombia/ ipc/ruiz.pdf

Runde, C. \& Flanagan, T. (2010). Developing your conflict competence: A handson guide for leaders, managers, facilitators, and teams (Vol. 152). New York: John Wiley y Sons.

Snodgrass, L. \& Blunt, R. (2009). The value of play for conflict management: A case study. South African Journal of Education, 29(1), 53-67. https://doi.org/10.1590/s0256-01002009000100004

Spillane, J. (2012). Distributed leadership (Vol. 4). New York: John Wiley y Sons. Stake, R. (1995). The art of case study research. New York: Sage Publications.

Stewart, B. (2015). Leading through the use of productive conflict: A professional development that teaches principals to use productive conflict among teachers for organizational learning and growth. (Dissertation for the degree of Doctor of Education) University of California, Berkeley, CA, USA. 
Taylor, S. y Bogdan, R. (1987). Introducción a los métodos cualitativos de investigación. Barcelona: Paidós.

Temperley, J. y Stoll, L. (2011). Mejorar el liderazgo escolar. Herramientas de Trabajo. Paris: OECD.

Touraine, A. (2006). Los movimientos sociales. Revista Colombiana de Sociología, 27(1), 255-278. Recuperado de

http://revistas.unal.edu.co/index.php/recs/article/view/7982/8626

Tschannen-Moran, M. (2014). Trust matters: Leadership for successful schools. New York: John Wiley y Sons.

Unline, C., Tschannen-Moran, M., \& Perez, L. (2003). Constructive conflict: How controversy can contribute to school improvement. Teachers College Record, 105(5), 782-815.

https://doi.org/10.1111/1467-9620.00268

Villalobos, C. (2016). El campo educativo en Chile post-dictadura (19902013). Continuidad y ruptura en la implementación del neoliberalismo en educación. En A. Pinol (Ed.), Democracia versus neoliberalismo. 25 años de neoliberalismo en Chile (pp. 159-178). Santiago de Chile: Fundación Rosa Luxemburgo-ICAL.

Villalobos, C. Peña, J. y Weinstein, J. (2016). Concepto de conflictos socioeducativos. Documento de Trabajo. Santiago de Chile: Centro de Liderazgo Educativo (CEDLE), Universidad Diego Portales.

Wagner, U. (2005). Konfliktforschung und Friedenssicherung. Psychologie in Gesellschaft, Kultur und Umwelt. Handbuch, 1, 55-60.

Weinstein. J. (2007). Chile 2000: La negociación Mineduc-Colegio de Profesores. Una visión personal. En V. Espínola y A. Accioly (Eds.), Sindicalismo docente y reforma educativa (pp. 59-108). Washington DC: Banco Interamericano de Desarrollo.

Weinstein, J., Cuellar, C., Hernández, M., y Flessa, J. (2015). Experiencias innovadoras y renovación de la formación directiva latinoamericana. Revista Iberoamericana de educación, 69(1), 23-46. Recuperado de http://rieoei.org/rie69a01.pdf

Yin, R. (1994). Case study research: Design and method (Third edition). Thousand Oaks: Sage Publications.

Recibido: 18/11/2016

Aceptado: 18/08/2017 